\title{
Détermination des choix alimentaires des brebis sur parcours steppique de Crau par une méthode d'étude de la végétation
}

\author{
D Hubert 1, T Adama 2, P Lapeyronie 1, G Molénat 1 \\ IINRA-ENSA.M, Unité de Zootechnie Méditerranéenne, 34060 Montpellier, France; \\ 2School of Agriculture, Fed Univ of Technology, PMB 65 Ninna, Nigéria
}

Pour étudier l'ingestion par un troupeau ovin au pâturage sur parcours, ainsi que la composition botanique du bol alimentaire, une lecture fine de la végétation a été testée sur un parcours amélioré de la Crau aux printemps 1993 et 1994 (50 à $69 \%$ de graminées, 9 à $21 \%$ de légumineuses, 1 à $9 \%$ de composées, 2 à $32 \%$ de matériel sec). Cette méthodologie associe la méthode des points quadrats (Daget et Poisonnet, 1971, An Agr, 22, 5-41) alignés (transects) avec une mesure de la hauteur maximale de chaque espèce (Heady, 1957, Ecology, 38, 313-320) en chaque point échantillonné. La végétation disparue, différence entre les mesures avant et après pâturage, est assimilée à la consommation par les animaux. Après des mises au point d'ordre méthodologique sur les lectures de végétation, quelques résultats sur les choix d'espèces consommées sont exposés.

II existe une relation linéaire $(r=0,89 ; n=20)$ entre les biomasses et la somme de toutes les hauteurs des espèces pour l'ensemble des mesures avant et après pâturage, ainsi qu'entre biomasse disparue et somme des hauteurs de végétation disparue $(r=0,97 ; n=$ 10). La contribution des espèces à la ration moyenne du troupeau peut être exprimée par leur contribution à la biomasse (CSB) ou bien par leur contribution à la hauteur $(\mathrm{CSH})$. La corrélation entre les CSB et les CSH est élevée $(r=0,94)$. Les quantités relatives des différentes espèces consommées peuvent donc être évaluées directement sur la végétation in situ. Lors de séquences de pâturage de 4 jours, avec un ajustement de l'offre alimentaire aux besoins des animaux, des mesures de consommation sur transects ont été effectuées à J0, J2 et $\mathrm{J} 4$ (début, milieu et fin de pâturage). On définit un $\mathrm{CUH}$ par espèce, par groupe d'espèces ou bien global (pour l'ensemble des espèces) :

CUH $=\frac{\text { Hauteur offerte }- \text { Hauteur refusée }}{\text { Hauteur offerte }}$

Ce coefficient permet d'établir une hiérarchie dans les préférences des animaux. Les espèces sont classées en quatre groupes : non recherchées $(0<\mathrm{CUH}<0,25)$, faiblement recherchées $(0,25<\mathrm{CUH}<0,50)$, moyennement recherchées $(0,50<\mathrm{CUH}<0,75)$, recherchées $(0,75<\mathrm{CUH}<1)$. Pour l'analyse des préférences alimentaires nous avons recours aux $\mathrm{CUH}$ calculés entre $0-2,2-4$ et $0-4$ jours.

Les légumineuses et les composées peu abondantes sont les plus recherchées. Parmi les graminées dominantes Dactylis hispanica et Dichanthium ischaemum sont recherchées de façon identique entre 0-2 jours et entre 2-4 jours, $(0,46$ et 0,38$)$ par contre les Vuipia sont refusées en fin de pâturage $(0,53$ et 0,04 respectivement).

\begin{tabular}{|c|c|c|c|c|}
\hline & $\mathrm{CSH}$ & CUH $0-4$ & $\mathrm{CUH} 0-2$ & $\mathrm{CUH} 2-4$ \\
\hline Graminées & 59 & 0,59 & 0,46 & 0,25 \\
\hline Légumineuses & 13 & 0,90 & 0,82 & 0,45 \\
\hline Composées & 5 & 0,87 & 0,74 & 0,47 \\
\hline Matériel sec & 13 & 0,56 & 0,43 & 0,23 \\
\hline
\end{tabular}

